\title{
Scoring of tumor-infiltrating lymphocytes: from visual estimation to machine learning
}

\author{
Klauschen F. ${ }^{1, *}$, Müller K.-R. ${ }^{2, *}$, Binder A. ${ }^{3}$, Bockmayr M. ${ }^{1,4}$, Hägele M. ${ }^{2}$, Seegerer P. ${ }^{2}$, Wienert S. ${ }^{1}$, \\ Pruneri G. ${ }^{5}$, de Maria S. ${ }^{6}$, Badve S. ${ }^{7}$, Michiels S. ${ }^{8}$, Nielsen T.O. ${ }^{9}$, Adams S. ${ }^{10}$, Savas P. ${ }^{11}$, Symmans F. ${ }^{12}$, \\ Willis S. ${ }^{13}$, Gruosso T. ${ }^{14}$, Park M. ${ }^{14}$, Haibe-Kains B. ${ }^{15,16,17,18,19}$, Gallas B. ${ }^{20}$, Thompson A.M. ${ }^{21}$, Cree I. ${ }^{22}$, \\ Sotiriou C. ${ }^{23}$, Hewitt S.M. ${ }^{24}$, Rimm D. ${ }^{25}$, Viale G. ${ }^{26}$, Loi S. $^{11}$, Loibl S. ${ }^{27}$, Salgado R. ${ }^{28}$, Denkert C. ${ }^{1 *}$ on \\ behalf of the International Immuno-Oncology Biomarker Working Group
}

1 Institute of Pathology, Charité Universitätsmedizin Berlin, Berlin, Germany 2 Machine Learning Group. Technical Universitv Berlin. Berlin, Germany, 3 Singapore University of Technology and Design (SUTD), Singapore 4 Department of Pediatrics, Universitätsklinikum Eppendorf, Hamburg, Germany 5 Department of Pathology, IRCCS Fondazione Istituto Nazionale Tumori and University of Milan, School of Medicine, Milan, Italy.

6 Service de Biostatistique et d'Epidémiologie, Gustave Roussy, B2 M, RdC,114 rue Edouard-Vaillant, 94805, Villejuif, France; CESP, Fac. de médecine - Univ. Paris-Sud, Fac. de médecine - UVSQ, INSERM, Université Paris-Saclay, Villejuif, 94805, France.

7 Department of Pathology and Laboratory Medicine, Indiana University, Indianapolis, USA. 8 Centre de Recherche en Epidémiologie et Santé des Populations, INSERM U1018, Service de Biostatistique et d'Epidémiologie, Gustave Roussv, Université Paris-Sud, Villeiuif, France.

9 Department of Pathology and Laboratory Medicine, Genetic Pathology Evaluation Centre, Universitv of British Columbia. Vancouver. Canada

10 Perlmutter Cancer Center. New York Universitv Medical School. New York. USA

11 Division of Research and Cancer Medicine, Peter MacCallum Cancer Centre, University of Melbourne, Victoria, Australia

12 Department of Pathologv. The UT M.D. Anderson Cancer Center. Houston. TX. USA.

13 Dept. of Molecular and Experimental Medicine, Avera Cancer Institute, Sioux Falls, SD, USA

14 Goodman Cancer Research Centre, McGill University, 1160 Pine Avenue West Rm511, Montreal, QC H3A 1A3, Canada

15 Princess Margaret Cancer Centre, University Health Network, Toronto, Canada

16 Medical Biophysics Department, University of Toronto, Toronto, Canada

17 Computer Science Department, University of Toronto, Toronto, Canada

18 Ontario Institute for Cancer Research, Toronto, Canada

19 Vector Institute, Toronto, Canada

20 US Food and Drug Administration, Center for Devices and Radiological Health, Office of Science and Engineering Laboratories, Division of Imaging, Diagnostics, and Software Reliability, Silver Spring, MD, USA

21 Department of Pathology, Ninewells Hospital \&Medical School, Dundee DD1 9SY, UK.

22 International Agency for Research on Cancer (IARC), World Health Organization, 150 Cours Albert Thomas, 69372 Lyon CEDEX 08, France

23 Department of Medical Oncology, Institut Jules Bordet, Université Libre de Bruxelles, Brussels. 24 Laboratory of Pathology, National Cancer Institute, National Institutes of Health, Bethesda MD, USA

25 Department of Pathology, Yale Schoole of Medicine, New Haven, CT, USA

26 Department of Pathology, Istituto Europeo di Oncologia, University of Milan, Milan, Italy.

27 German Breast Group, Neu-Isenburg, Germany

28 Breast Cancer Translational Research Laboratory/Breast International Group, Institut Jules Bordet, Brussels Department of Pathology and TCRU, GZA, Antwerp, Belgium.

*corresponding authors

This is the author's manuscript of the article published in final edited form as:

Klauschen, F., Müller, K.-R., Binder, A., Bockmayr, M., Hägele, M., Seegerer, P., ... Denkert, C. (2018). Scoring of tumorinfiltrating lymphocytes: From visual estimation to machine learning. Seminars in Cancer Biology, 52, $151-157$. 


\begin{abstract}
The extent of tumor-infiltrating lymphocytes (TILs), along with immunomodulatory ligands, tumormutational burden and other biomarkers, has been demonstrated to be a marker of response to immune-checkpoint therapy in several cancers. Pathologists have therefore started to devise standardized visual approaches to quantify TILs for therapy prediction. However, despite successful standardization efforts visual TIL estimation is slow, with limited precision and lacks the ability to evaluate more complex properties such as TIL distribution patterns. Therefore, computational image analysis approaches are needed to provide standardized and efficient TIL quantification. Here, we discuss different automated TIL scoring approaches ranging from classical image segmentation, where cell boundaries are identified and the resulting objects classified according to shape properties, to machine learning-based approaches that directly classify cells without segmentation but rely on large amounts of training data. In contrast to conventional machine learning (ML) approaches that are often criticized for their "black-box" characteristics, we also discuss explainable machine learning. Such approaches render ML results interpretable and explain the computational decision-making process through high-resolution heatmaps that highlight TILs and cancer cells and therefore allow for quantification and plausibility checks in biomedical research and diagnostics.
\end{abstract}

\title{
Introduction
}

Computational image analysis in histopathology has been around for several decades[1, 2]. However, its application in diagnostics has been limited, in part due to the very limited capabilities of computer hardware restricting slide digitization, processing and analysis as well as storage. In addition, qualitative or semi-quantitative visual evaluation mostly sufficed as clinical decision-making was not refined to a point where quantitative diagnostic results were required. In recent years, digital pathology has received increasing attention because whole slide scanning capabilities and desktop computer hardware capable of processing and displaying whole slide images at high resolution have become widely available. Moreover, increasingly refined biomarker-based patient stratification calls for accurate quantitative evaluation of the relevant histological and molecular properties[3]. Particularly because molecular profiling provides allegedly precise numeric results without any interrater reliability issues, demands for histopathological evaluation including morphological and immunohistochemical evaluation to keep up with these developments of quantitative diagnostics are greater than ever. Therefore, systematic manual and computational pathology strategies have been proposed to facilitate the quantitative evaluation of both morphological and immunological markers, which will be reviewed here.

Prominent examples of increased demands for quantitative histopathological evaluation include proliferation scoring in breast and other cancers based on Ki67-immunhistochemistry[4] and, more recently, immuno-oncology. Here, tumor-infiltrating lymphocytes (TILs) scored on Hematoxylin\&Eosin (H\&E) or immunohistochemistry (IHC) slides have been shown to be not only prognostically, but also therapeutically relevant in breast cancer, non-small cell lung cancer and melanoma[5-7]. Administration of immune-checkpoint inhibitors is becoming an important part of 
tumor treatment for an increasing number of tumor entities, but its therapeutic success is dependent on several parameters such as the expression of immunomodulatory ligands (such as PD-L1[8]), tumor mutational burden and, last but not least, the immune cell infiltrate[9, 10].

Currently, the most prominent example where TIL scoring is clinically relevant is breast cancer. The relevance of immunological parameters for chemotherapy response[11] in triple-negative breast cancer has led to phase 1 and phase 2 trials investigating immune checkpoint inhibitors in this subset of tumors. These trials have reported objective response rates (ORR) of 5-23\%[12-14] for immune checkpoint inhibitor monotherapy in the metastatic setting depending on the line of therapy. Response rates can be increased for immune checkpoint inhibitors when given in combination with chemotherapy, for example a chemo-immunotherapy trial reported ORR of $38 \%$ for the combination in the metastatic setting (54\% in front-line) [15]. Importantly, as for immune checkpoint monotherapy, line of therapy was important, with highest ORR in the front-line setting, and responses appeared durable[16]. At the 2017 ASCO meeting, a neoadjuvant trial[17] of a combination of immune checkpoint inhibitors and chemotherapy has reported increased pathological complete response (PCR) rates in triple-negative tumors, but also in luminal breast cancer. Because of the observation made for many cancers that while only a minority of tumors responds to therapy, those that do respond typically have a substantially improved prognosis[18-20] and because the therapies involved are expensive and can have serious toxicities, analytically - and clinically - valid predictive biomarkers are critically needed. PD-L1 expression in TNBC has been shown to enrich for responses in some but not all trials[13, 14], however, preliminary data of TIL as predictive biomarker appear promising in metastatic TNBC[21]. As diverse predictors of therapy response have been proposed, combinations of quantitative molecular and spatio-morphological parameters should be explored for an improved understanding of the biology of tumor-immune-cell interactions and the identification of predictive markers.

Over the last couple years, different strategies for developing clinically applicable immune biomarkers have been proposed. One widely-adopted current diagnostic approach to evaluate tumorinfiltrating lymphocytes (TILS) on standard H\&E sections has been described in guidelines published by the International TIL working group. Briefly, TILs are divided into intra-tumoral and stromal TILs that are quantitated separately, but any additional spatial distribution patterns are not assessed due to a lack of standardized methodology, time and difficulty in making such assessments. The international TIL Guidelines are made for visual evaluation of H\&E sections by pathologists[22, 23] and their robustness has been shown in international ring trials[24]. Quantification of TILs has the potential to become a new tumor biomarker as an easy approach to evaluate the immunogenicity of a tumor.

However, despite these successful standardization efforts, visual assessment requires extensive training by pathologists and inter-observer variability will likely remain an issue given the difficulty in quantitatively evaluating histological properties. Automated image analysis methods can help improve quantification accuracy, save time and facilitate the analysis of more complex spatial patterns and provide standardized metrics for rigorous validation by expert pathologists and quality assurance through regulatory agencies.

While TIL scoring in conventional H\&E sections has the advantage that no additional stains are required, lymphocyte subtyping into cytotoxic, helper, regulatory and other T cells not possible in H\&E may become clinically relevant in the future[25]. Therefore, in addition to TIL detection through morphological (H\&E) features, subtyping of TILs through immunohistochemistry or more advanced 
multiplex imaging approaches may become necessary and will also require adequate image analysis strategies.

In this review, we will discuss computational pathology approaches to TIL scoring with a focus on machine learning strategies. Although a broad variety of computational image analysis approaches exists, they can be grouped into segmentation and direct classification methods (Fig. 1). While the techniques may show some overlap, segmentation primarily refers to approaches that first identify shapes and distinct "segments" within images which are then classified in a second step. Direct classification refers to machine learning methods that identify objects of interest in images without explicit selection of distinct image shapes [26].

\section{Classical image segmentation for TIL detection}

Classical segmentation methods are unsupervised or semi-supervised and require small amounts of training data. Such methods include threshold or watershed-segmentation, morphology-based segmentation, level set methods, color-space clustering-based approaches [27-30] as well as more advanced approaches such as deformable models[31] or metaheuristic-based methods[32]. In general, segmentation-based image analysis includes three different aspects. First, the raw segmentation process normally identifies edges within the image and thereby subdivides the image into (disjunct) "segments". With reference to approaches described below, this step also occurs implicitly in deep learning-based segmentation such as the approach by Chen et al. [33]. It was shown that lower layers of convolutional neural networks learn to represent edges[34]. Second, this approach identifies image segments that are regarded as target objects in the context of the application based on pre-defined parameters (i. e. identification of cells in histological images). Finally, features are extracted and target objects are classified into lymphocytes, fibroblasts and cancer cells. This can be done with any type of classification approach from simple parameter range selection such as cell size or shape features to more complex machine learning techniques.

An advantage of object detection by classical segmentation is that it requires a limited training set. However, the disadvantage is that parameters need to be provided based on a priori knowledge and that segmentation results are sensitive to small changes in biological and technical image variation. Moreover, the downstream classification accuracy of segmented objects is highly dependent on the prior segmentation results which can cause over-fitting and weak performance with external datasets. To reduce the limitations due to requirements of a priori knowledge, Wienert et al. proposed a minimum-model segmentation approach which minimizes necessary parameters provided $a$ priori $[35,36]$. However, while this approach can be adapted easily to the identification of lymphocytes which have little morphological variability, the classification of the highly variable cancer cells and their differentiation from normal cells such as gland and duct epithelia or even stromal cells based on predefined parameters is difficult. Quantification of lymphocytes without the identification of carcinoma cells ignores their spatial relationship, but this information may indeed be relevant in the clinical setting, because of the typically heterogeneous distribution of TILs. In breast cancer, the majority of TILs are located in the tumor stroma, while only a minority of lymphocytes have direct contact with tumor cells. The current international guidelines recommend focusing on stromal TILs as a main parameter[37] which recognizes the challenges faced by clinical pathologists in reviewing whole slide images.

However, scoring immune cell abundance in the stroma may not be sufficient in all cases. In a recent study, Yuan and coworkers use a segmentation-classification approach to show that spatial 
clustering was associated with poor recurrence-free survival after endocrine therapy in ER+ breast cancer whereas immune cell abundance had no prognostic effect[38]. This study also demonstrates that computational image analysis not only allows for automated quantification of tumor-infiltrating lymphocytes, but also facilitates the evaluation of the clinical relevance of more complex tissue properties such as spatial patterns of TIL distribution.

Despite such successful applications of segmentation-based approaches, a general critique of segmentation approaches is that they complicate the cell identification process by introducing an intermediate segmentation step instead of directly classifying objects or predicting the quantities of interest[26]. More specifically, for detecting and quantifying TILs it is sufficient to identify the approximate cell coordinates and not necessary to detect their precise cell boundaries as is done in segmentation. Therefore, relying on segmentation, one resorts to solving a more complex problem than actually needed, and inaccuracies from the segmentation process impact the intended prediction for TIL quantities. Direct classification of cells through machine learning-based image analysis approaches may improve performance which will be described in the following sections.

\section{Machine learning-based classification}

The term "machine learning" refers to mathematically diverse approaches such as, for instance, support vector machines (SVM)[39] or deep neural networks (dNN)[40] to solve prediction problems including classification, detection and segmentation. The learning machines, which are implementations of classification algorithms, are trained using large sets of (manually annotated) data and thereby acquire the ability to predict out-of-sample objects (i.e. the machine was trained to learn the properties of the class the object belongs to, but the particular object was not part of the training cohort). Machine learning approaches have been proposed for a large variety of histological image analysis tasks including, but not limited to, mitosis counting, nucleus detection or identification of tissue components (cancer vs normal cells), lymphoma subtyping and lymphocyte detection[41]. Stateof-the-art deep neural networks typically use an input image or tile for training and classification of around $300 \times 300$ pixels. Larger sizes are possible, in principle, but the size of the input image is constrained primarily by the memory of the GPUs (graphics processing unit) necessary to fit the large number of feature maps present in deep neural network models. Histological whole slide images at $40 x$ magnification have sizes of up to $100,000 \times 150,000$ pixels which adds to the complexity of deep learning approaches.

Machine learning-based approaches that can offer segmentation rely on conditional random fields[42] or deep learning models such as the U-Net[43] or fully convolutional neural nets [44]. Combinations of above ideas such as the Deeplab-CRF [33] and derivatives thereof have been successfully applied to histopathology [45]. Another fully convolutional network (FCN) approach is capable of detecting lymphocytes but also other cell types with location information [46]. Achieving high quality (segmentation) results with this group of methods requires large scale datasets for training (tens of thousands of cells) with precisely annotated segmentation masks accurately representing the cell borders or the exact locations of all cells of the relevant types in training images, which is a tremendous manual annotation effort and prone to variation.

Other machine learning approaches offer alternatives to segmentation through object detection which output bounding boxes around object of interest (cells). These approaches are wellestablished in computer vision for the detection of man-made objects or human body parts; examples are Fast R-CNN[47], Faster R-CNN[48] and YOLO[49]. However, applying these techniques for 
identification of cell types, including lymphocytes, may be much more challenging than to man-made objects, as histomorphological variability is high (particularly in cancer). Moreover, it is often insufficient to classify a single cell without accounting for contextual information. The cell recognition process may substantially benefit from using context by looking at the neighborhood of a cell, as done in the dNN-based segmentation approaches mentioned before. However, this comes at the cost of more complex annotations consisting of precise cell boundaries.

Here, machine learning-based image classification approaches that directly identify cells without object segmentation may be more efficient. Although these classification-based approaches require neither a priori parameters defining the objects to be classified nor precise segmentation masks for the training data, training is still a critical component due to the need for weight learning in neural networks. Images are sub-tiled into regions and then image tiles are classified with respect to the presence or absence of cell types. Depending on the complexity of the task at hand, hundreds to thousands of image tiles containing the relevant cell types are needed and the composition of the training data set critically influences the classification results. Therefore, a balanced unbiased and representative training data set is essential for a good generalization of the machine learning algorithm to out-of-sample data. While lymphocytes display relatively little morphological variation, training for tumor cell classification is more complex because of the large morphological variability of cancer cells. Classification results also depend on technical parameters such as the tile size used for training and classification. Image tiles that are large relative to the cell sizes allow for the use of contextual information that may often be relevant for classification. However, while machine learning algorithms are robust to moderate amounts of label noise, information on the contained cell types may be "diluted" if - for a too large fraction of training data - more than one cell type is present (which is normally the case for image regions large compared to the size of a single cell). On the other hand, using tiles of the size of single cells may be problematic due to a large range of cell sizes from pleomorphic tumor cells to lymphocytes apart from the fact that potentially important contextual information is ignored. Most current machine learning approaches provide classification results with a resolution limited to the tile sizes used during training. Their advantage is a relatively easy annotation that requires only the information whether an image patch contains a cell of interest or not in contrast to precise annotations of cell shapes, bounding boxes or cell centers.

Several approaches for leukocyte/lymphocyte quantification using machine learning have been proposed recently. Zhao et al. detect all major subtypes of leukocytes including lymphocytes in peripheral blood using convolutional neural networks[50]. However, approaches tested for blood smears, which are less complex than histological images, may not generalize to TIL detection in cancer tissue specimens. Chen et al. [51] propose a deep neural network approach that combines approximate manual labeling of individual cells with prior knowledge about the uniformity of lymphocyte size and shape to reduce the training data annotation effort. While designed for application in tissue images, their approach is optimized for lymphocyte detection and does not necessarily generalize well to tumor cell detection. Similar assumptions were made for the training data annotation in the segmentation stage of the approach presented in [46], where all positions of cell nuclei were labeled. This work is based on a fully convolutional network for segmenting nuclei independent of type with a subsequent classification of small windows around the detected cells. Similarly, Garcia et al., 2017 [52] and Chen and Chefd'hotel [53] propose deep convolutional neural networks to identify lymphocytes in immunohistological images. Swiderska-Chadaij et al. [54] 
compare different patch classification, bounding box detection and segmentation algorithms for immunohistochemically stained whole-slide images. The TIL detection capabilities in these three works rely, however, on $T$ lymphocyte-specific markers (CD3, CD8) to facilitate the discrimination against background, which is substantially less complex than lymphocyte detection in H\&E stained histomorphological images.

\section{Interpretable machine learning}

Interpretability of classical machine learning approaches for image analysis is normally limited by the resolution defined by the size of the image patches used for training. Location information is usually achieved by using patch sizes similar to that of cells. For different cell types, such as small lymphocytes and larger tumor cells, using dual tile sizes can be an option. A sliding window is moved over a larger image. If the scan process "hits" a relevant cell, location information is gained, which can be used to generate probability heatmaps showing cell locations[46], where the heatmap resolution depends on size and overlap of tiles. However, if contextual information around cells matters, patch sizes need to be large relative to cell sizes and with a classification score for each tile, classification results have a relatively coarse resolution not allowing the image analyst (pathologist) to understand and check the plausibility of the classification result (Figure 2). This fact in combination with the complexity of the underlying models has led to the criticism that machine learning is a "black-box" technique, which limits its acceptance and is particularly critical in medicine, where physicians are required to carefully check the plausibility of the diagnostic results. Even in a possible future fully-automated classification approach, the results still need to be reviewed by experienced pathologists. Here, so-called explainable machine-learning approaches offer a solution as explained below.

Several approaches have been developed to explain machine learning predictions, such as Sensitivity, Guided Backprop, LIME, deepLIFT, integrated Gradients or layer-wise relevance propagation (LRP, see below). Although they all share the property that they take a prediction for an image as input and compute as output scores (often rendered as a heatmap) for regions or pixels of the input image attempting to explain the prediction decision, the methods differ substantially. The well-known gradient-based methods, for instance, explain which regions are most sensitive to changes in the input, which are often different from those regions which contribute most to a prediction.

The recently developed "layer-wise relevance propagation" (LRP) $[55,56])$ traces back the contributions of image regions to the classification result and computes pixel-wise scores based on a decomposition principle. The results can be rendered in heatmaps that visualize the classification result and allow for plausibility checks by overlaying original image with heatmap information (Figure 2 right panel). The advantage of LRP over the heatmaps obtained by patch-wise classification described above is that it is capable of taking larger context into account for the prediction without reducing its capacity to provide spatially highly resolved results. Although fully convolutional neural network approaches can also provide high resolution (heatmap) results, they require detailed annotations for cell shapes and locations which are cumbersome to obtain and subject to inter- and intra-rater variability. In contrast to that, LRP neither requires segmentation labels nor cell locations for training. Instead the approach relies on only relatively weak supervision on the patch level, e. g. labels indicating the presence of at least one lymphocyte or tumor cell. In summary, LRP works with image tiles relatively large compared to the objects of interest (i. e. cancer cells, lymphocytes), but outputs high resolution heatmaps showing the (pixel-wise) classification scores. These heatmaps allow for an accurate localization and quantification of lymphocytes and other cell types and even provide cell 
shape information (Figure 2). LRP is therefore particularly useful when studying data with strong variability with the need to include contextual information and for efficient training data annotation and result explanation[57]. LRP implementations have also been shown to work with large and very deep neural networks such VGG [58] and DenseNets [59] (cf. [60] and https://github.com/albermax/innvestigate for a comparison of different explanation methods).

\section{Performance evaluation and quality assurance in machine learning}

Although machine-learning based image analysis shows less sensitivity towards cellular parameters defined a priori as in conventional segmentation as described above, performance depends on available training data and technical properties of the ML approach. Therefore, systematic performance evaluation and quality assurance standards with benchmark image data sets should to be defined and implemented prior to clinical application of machine learning for TIL scoring similar to what has been done for microarray data[61].

\section{Estimating tumor-infiltrating lymphocytes from molecular data.}

Besides image-analysis based methods, machine learning-based deconvolution approaches have been proposed to estimate the amount and the composition of tumor-infiltrating immune cells from molecular profiles. These methods are attractive since they quantify several immune cell populations simultaneously, however, they do not allow for a spatial localization of infiltrating immune cells. Moreover, the required molecular profiles (mostly gene expression) are in general not available in routine diagnostics. As an example, CIBERSORT, one of the most widely used methods, applies support vector regression to quantify 22 hematopoietic subpopulations from bulk gene expression data[62]. The more recently proposed Microenvironment Cell Populations-counter (MCPcounter) uses specific gene expression signatures that allow for the robust quantification of 8 immune cell populations and 2 stromal cell populations[63]. These methods have been successfully used to infer proportions or absolute levels of immune cell populations and their prognostic relevance in large datasets including several different cancer-types[63-65]. They are also attractive to study tumor-associated immune cells in rare cancer types, where large image-based studies are unfeasible due to sample size restrictions (for immunohistochemistry) or where TILs cannot be reliably detected on HE slides, e.g. in small-roundblue-cell tumors [66] (Bockmayr et al, Oncoimmunology, 2018, in press).

Additionally, omics based approaches for the characterization of immune infiltrates have also been proposed. For example, a methylation signature that allows for the quantification of TILs in breast cancer and other tumors has been recently published[67] and another study relies on multi-omics data to identify cytokines to be predictive of TIL levels [68].

\section{Integration of machine learning-based image analysis with molecular information}

Machine learning-based approaches may also contribute to immuno-oncology by integrating imagebased quantification of TILs, immune cell enrichment analyses corresponding to those described above and analyses of local structures in TIL patterns. Yuan et al.[69] showed that an integrated predictor of survival in estrogen receptor negative breast cancer combing TIL scores from image based analysis and gene expression signatures outperforms classifiers that use only a single data type. Saltz et al. present an analysis based on 13 TCGA tumor types [70] and demonstrate that differential morphological patterns of TIL distributions exist across cancers which may hint to distinct spatial relations between 
different immune cell types in the context of different tumor types and molecular backgrounds. In addition to such correlative analysis, we recently developed an approach that extends layer-wise relevance propagation (LRP) beyond histological classification to predicting molecular tumor properties from morphological tumor properties available through H\&E stains which is the computational basis for an integrative, morpho-molecular pathology[57].

\section{Outlook}

The present studies on TIL analysis illustrate that computational pathology, and in particular, machine learning based approaches, will increasingly contribute to a better understanding of histopathological images and the relation of spatio-morphological properties, molecular alterations and the immune system in cancer with applications in research and diagnostics. Their main contribution for the foreseeable future will, however, not be in replacing the pathologist in rendering diagnoses, where the (qualitative) decision if a tissue alteration is benign or malignant still is the basis of all oncological therapies, but will assist the pathologist in more objectively quantifying additional parameters such as the amount and distribution of tumor-infiltrating lymphocytes. With the increasing relevance of "omics" analyses including mutational profiling, epigenetics, gene and protein expression, machine learning will enhance and make histology "fit" for the age of precision medicine[71].

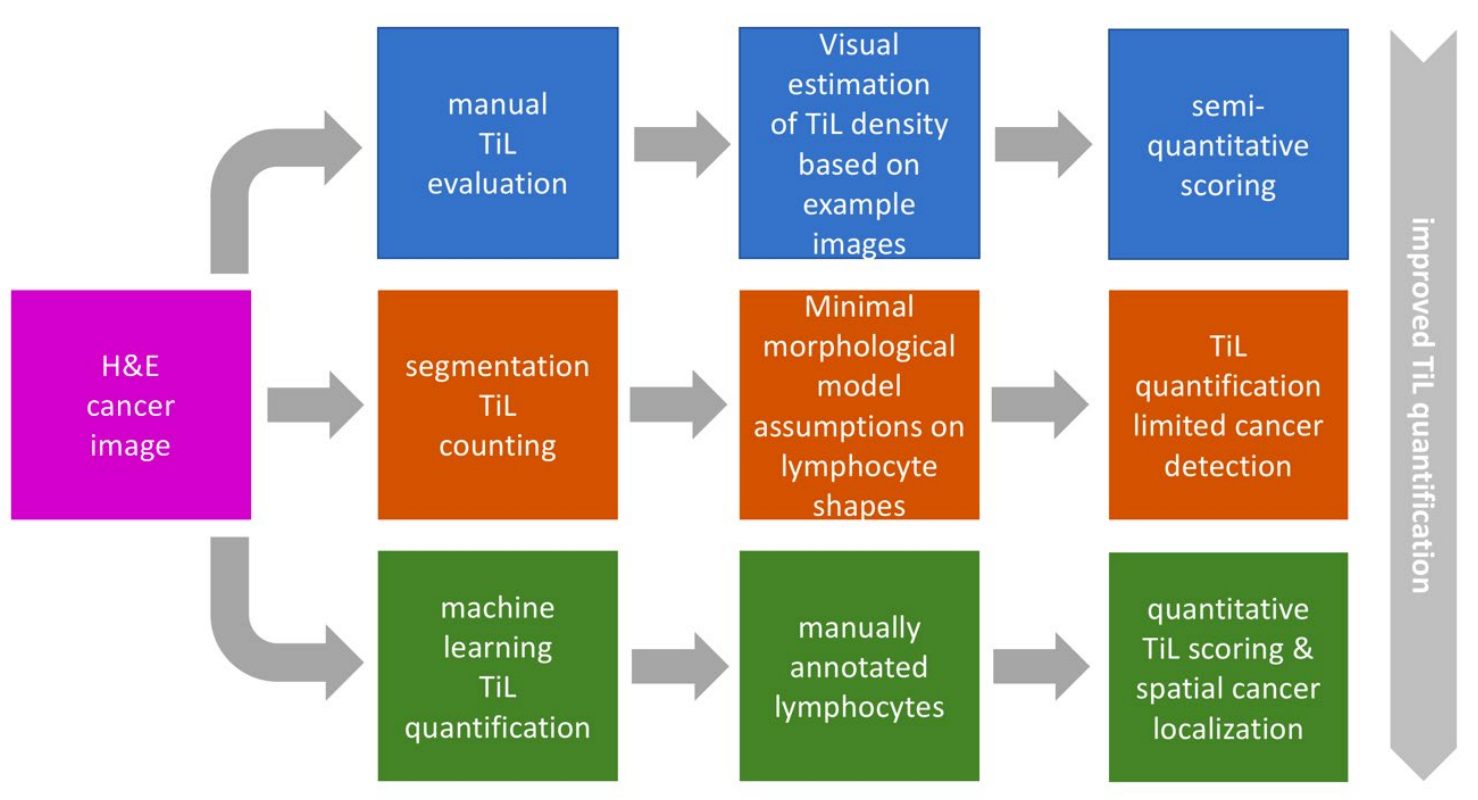

Figure 1: Different approaches of TIL evaluation. Top: classical evaluation of TILs is based on estimation of lymphocyte density, for instance, by providing the pathologist with example or simulated images for which quantification are known. Middle: Classical segmentation-based approaches rely on pre-defined parameters to model lymphocytes making them susceptible to image variability and often do not perform well at cancer cell detection. Bottom: machine learning approaches require large training data sets but allow for more efficient detection of lymphocytes and cancer cells than segmentation. 


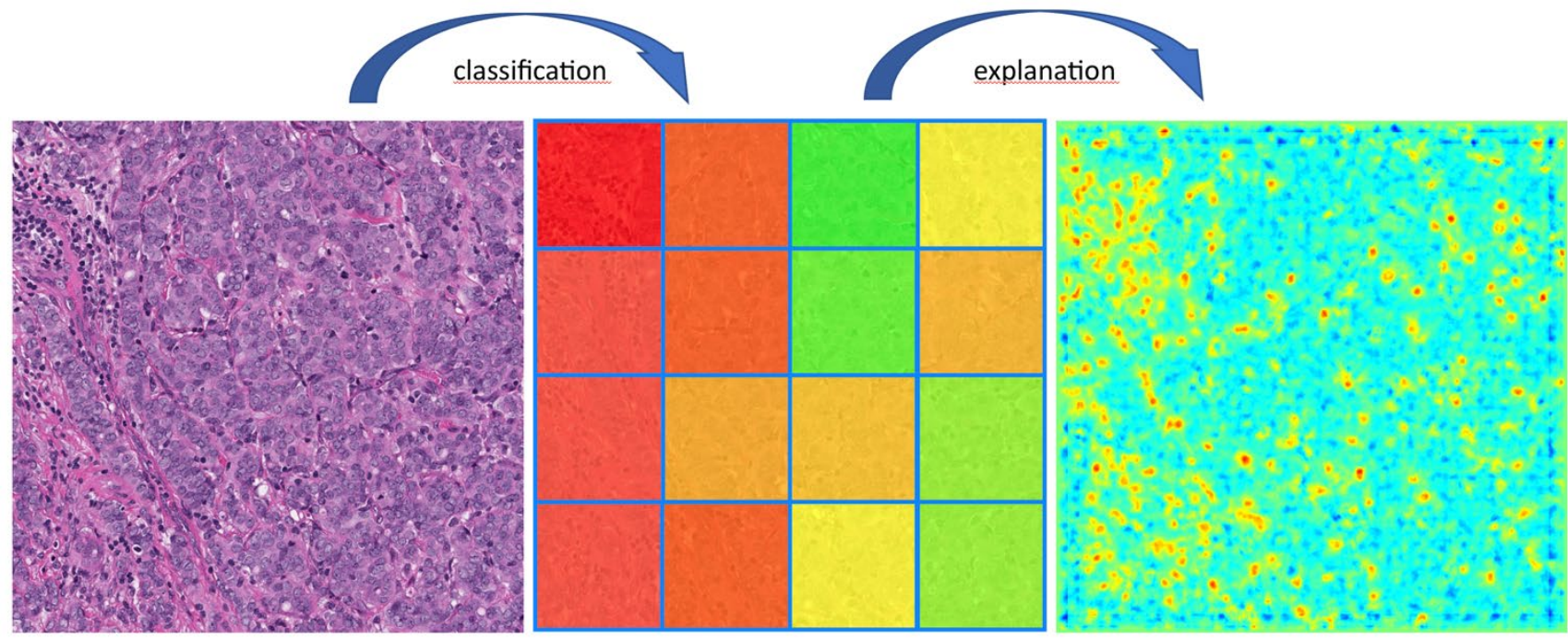

Figure 2: Machine-learning-based TIL evaluation. Left: original H\&E breast cancer image. Center: machine-learning-based classification/scoring of regions with respect to their lymphocyte content (green: no lymphocytes, red: high content of lymphocytes). Right: Heatmap explaining classifier decisions with pixel-wise resolution demonstrates how lymphocytes are identified and localized based on layer-wise-relevance propagation LRP. Lymphocyte counts can be derived from the heatmap or directly during classification (blue to green: no evidence of lymphocytes; yellow to red: increasing evidence of lymphocytes).

\section{References}

[1] S.H. Ong, X.C. Jin, Jayasooriah, R. Sinniah, Image analysis of tissue sections, Comput Biol Med 26(3) (1996) 269-79.

[2] E. Bengtsson, The measuring of cell features, Anal Quant Cytol Histol 9(3) (1987) 212-7.

[3] T.J. Fuchs, J.M. Buhmann, Computational pathology: challenges and promises for tissue analysis, Comput Med Imaging Graph 35(7-8) (2011) 515-30.

[4] F. Klauschen, S. Wienert, W.D. Schmitt, S. Loibl, B. Gerber, J.U. Blohmer, J. Huober, T. Rudiger, E. Erbstosser, K. Mehta, B. Lederer, M. Dietel, C. Denkert, G. von Minckwitz, Standardized Ki67 Diagnostics Using Automated Scoring--Clinical Validation in the GeparTrio Breast Cancer Study, Clin Cancer Res 21(16) (2015) 3651-7.

[5] S.M. Mahmoud, E.C. Paish, D.G. Powe, R.D. Macmillan, M.J. Grainge, A.H. Lee, I.O. Ellis, A.R. Green, Tumor-infiltrating CD8+ lymphocytes predict clinical outcome in breast cancer, J Clin Oncol 29(15) (2011) 1949-55.

[6] A. Uryvaev, M. Passhak, D. Hershkovits, E. Sabo, G. Bar-Sela, The role of tumor-infiltrating lymphocytes (TILS) as a predictive biomarker of response to anti-PD1 therapy in patients with metastatic non-small cell lung cancer or metastatic melanoma, Med Oncol 35(3) (2018) 25.

[7] M. Rakaee, T.K. Kilvaer, S.M. Dalen, E. Richardsen, E.E. Paulsen, S.M. Hald, S. Al-Saad, S. Andersen, T. Donnem, R.M. Bremnes, L.T. Busund, Evaluation of tumor-infiltrating lymphocytes using routine $H \& E$ slides predicts patient survival in resected non-small cell lung cancer, Hum Pathol (2018).

[8] H.M. Kluger, C.R. Zito, G. Turcu, M.K. Baine, H. Zhang, A. Adeniran, M. Sznol, D.L. Rimm, Y. Kluger, L. Chen, J.V. Cohen, L.B. Jilaveanu, PD-L1 Studies Across Tumor Types, Its 
Differential Expression and Predictive Value in Patients Treated with Immune Checkpoint Inhibitors, Clin Cancer Res 23(15) (2017) 4270-4279.

[9] B. Ingold Heppner, M. Untch, C. Denkert, B.M. Pfitzner, B. Lederer, W. Schmitt, H. Eidtmann, P.A. Fasching, H. Tesch, C. Solbach, M. Rezai, D.M. Zahm, F. Holms, M. Glados, P. Krabisch, E. Heck, A. Ober, P. Lorenz, K. Diebold, J.O. Habeck, S. Loibl, Tumor-Infiltrating Lymphocytes: A Predictive and Prognostic Biomarker in Neoadjuvant-Treated HER2-Positive Breast Cancer, Clin Cancer Res 22(23) (2016) 5747-5754.

[10] G.T. Gibney, L.M. Weiner, M.B. Atkins, Predictive biomarkers for checkpoint inhibitorbased immunotherapy, Lancet Oncol 17(12) (2016) e542-e551.

[11] L. Zitvogel, O. Kepp, G. Kroemer, Immune parameters affecting the efficacy of chemotherapeutic regimens, Nat Rev Clin Oncol 8(3) (2011) 151-60.

[12] R. Nanda, L.Q. Chow, E.C. Dees, R. Berger, S. Gupta, R. Geva, L. Pusztai, K. Pathiraja, G. Aktan, J.D. Cheng, V. Karantza, L. Buisseret, Pembrolizumab in Patients With Advanced Triple-Negative Breast Cancer: Phase Ib KEYNOTE-012 Study, J Clin Oncol 34(21) (2016) 24607.

[13] S. Adams, S. Loi, D.L. Toppmeyer, D.W. Cescon, M. De Laurentiis, R. Nanda, E.P. Winer, H. Mukai, K. Tamura, A.C. Armstrong, M.C. Liu, H. Iwata, L. Ryvo, P. Wimberger, H.S. Rugo, A. Tan, C. D'Aquanno, Y. Ding, V. Karantza, P. Schmid, Abstract PD6-10: KEYNOTE-086 cohort B: Pembrolizumab monotherapy for PD-L1-positive, previously untreated, metastatic triplenegative breast cancer (mTNBC), 2018.

[14] P. Schmid, C. Cruz, F.S. Braiteh, J.P. Eder, S. Tolaney, I. Kuter, R. Nanda, C. Chung, P. Cassier, J.-P. Delord, M. Gordon, Y. Li, B. Liu, C. O’Hear, M. Fasso, L. Molinero, L.A. Emens, Abstract 2986: Atezolizumab in metastatic TNBC (mTNBC): Long-term clinical outcomes and biomarker analyses, Cancer Research 77(13 Supplement) (2017) 2986-2986.

[15] S. Adams, R.D. Diamond, E.P. Hamilton, P.R. Pohlmann, S.M. Tolaney, L. Molinero, X. He, D. Waterkamp, R.P. Funke, J.D. Powderly, Phase $\mathrm{lb}$ trial of atezolizumab in combination with nab-paclitaxel in patients with metastatic triple-negative breast cancer (mTNBC). J Clin Oncol (ASCO) (2016) (suppl; abstr 1009).

[16] P.R. Pohlmann, D. JR, H. EP, S. Tolaney, W. Zhang, R.P. Funke, A. S, Atezolizumab + Nabpaclitaxel in Metastatic Triple-Negative Breast Cancer: 2-Year Update From a Phase Ib Trial, Proc Am Assoc Cancer Res. 2018;59: Abstract CT028 (2018).

[17] R. Nanda, M.C. Liu, C. Yau, S. Asare, N. Hylton, L. Van't Veer, J. Perlmutter, A.M. Wallace, A.J. Chien, A. Forero-Torres, E. Ellis, H. Han, A. Sanders Clark, K.S. Albain, J.C. Boughey, A.D.

Elias, D.A. Berry, D. Yee, A. DeMichele, L. Esserman, Pembrolizumab plus standard neoadjuvant therapy for high-risk breast cancer (BC): Results from I-SPY 2., J Clin Oncol ASCO Annual Meeting((suppl; abstr 506)) (2017).

[18] T. Powles, J.P. Eder, G.D. Fine, F.S. Braiteh, Y. Loriot, C. Cruz, J. Bellmunt, H.A. Burris, D.P. Petrylak, S.L. Teng, X. Shen, Z. Boyd, P.S. Hegde, D.S. Chen, N.J. Vogelzang, MPDL3280A (anti-PD-L1) treatment leads to clinical activity in metastatic bladder cancer, Nature 515(7528) (2014) 558-62.

[19] D. Schadendorf, F.S. Hodi, C. Robert, J.S. Weber, K. Margolin, O. Hamid, D. Patt, T.T. Chen, D.M. Berman, J.D. Wolchok, Pooled Analysis of Long-Term Survival Data From Phase II and Phase III Trials of Ipilimumab in Unresectable or Metastatic Melanoma, J Clin Oncol 33(17) (2015) 1889-94.

[20] P. Sharma, J.P. Allison, The future of immune checkpoint therapy, Science 348(6230) (2015) 56-61.

[21] S. Loi, S. Adams, P. Schmid, J. Cortés, D.W. Cescon, E.P. Winer, D.L. Toppmeyer, H.S. Rugo, M. De Laurentiis, R. Nanda, H. Iwata, A. Awada, A. Tan, A. Wang, G. Aktan, V. Karantza, 
R. Salgado, LBA13Relationship between tumor infiltrating lymphocyte (TIL) levels and response to pembrolizumab (pembro) in metastatic triple-negative breast cancer (mTNBC): Results from KEYNOTE-086, Annals of Oncology 28(suppl_5) (2017) mdx440.005$\mathrm{mdx} 440.005$.

[22] S. Hendry, R. Salgado, T. Gevaert, P.A. Russell, T. John, B. Thapa, M. Christie, K. van de Vijver, M.V. Estrada, P.I. Gonzalez-Ericsson, M. Sanders, B. Solomon, C. Solinas, G. Van den Eynden, Y. Allory, M. Preusser, J. Hainfellner, G. Pruneri, A. Vingiani, S. Demaria, F. Symmans, P. Nuciforo, L. Comerma, E.A. Thompson, S. Lakhani, S.R. Kim, S. Schnitt, C. Colpaert, C. Sotiriou, S.J. Scherer, M. Ignatiadis, S. Badve, R.H. Pierce, G. Viale, N. Sirtaine, F. PenaultLlorca, T. Sugie, S. Fineberg, S. Paik, A. Srinivasan, A. Richardson, Y. Wang, E. Chmielik, J. Brock, D.B. Johnson, J. Balko, S. Wienert, V. Bossuyt, S. Michiels, N. Ternes, N. Burchardi, S.J. Luen, P. Savas, F. Klauschen, P.H. Watson, B.H. Nelson, C. Criscitiello, S. O'Toole, D. Larsimont, R. de Wind, G. Curigliano, F. Andre, M. Lacroix-Triki, M. van de Vijver, F. Rojo, G. Floris, S. Bedri, J. Sparano, D. Rimm, T. Nielsen, Z. Kos, S. Hewitt, B. Singh, G. Farshid, S. Loibl, K.H. Allison, N. Tung, S. Adams, K. Willard-Gallo, H.M. Horlings, L. Gandhi, A. Moreira, F. Hirsch, M.V. Dieci, M. Urbanowicz, I. Brcic, K. Korski, F. Gaire, H. Koeppen, A. Lo, J. Giltnane, M.C. Rebelatto, K.E. Steele, J. Zha, K. Emancipator, J.W. Juco, C. Denkert, J. Reis-Filho, S. Loi, S.B. Fox, Assessing Tumor-Infiltrating Lymphocytes in Solid Tumors: A Practical Review for Pathologists and Proposal for a Standardized Method from the International ImmunoOncology Biomarkers Working Group: Part 2: TILs in Melanoma, Gastrointestinal Tract Carcinomas, Non-Small Cell Lung Carcinoma and Mesothelioma, Endometrial and Ovarian Carcinomas, Squamous Cell Carcinoma of the Head and Neck, Genitourinary Carcinomas, and Primary Brain Tumors, Adv Anat Pathol 24(6) (2017) 311-335.

[23] S. Hendry, R. Salgado, T. Gevaert, P.A. Russell, T. John, B. Thapa, M. Christie, K. van de Vijver, M.V. Estrada, P.I. Gonzalez-Ericsson, M. Sanders, B. Solomon, C. Solinas, G. Van den Eynden, Y. Allory, M. Preusser, J. Hainfellner, G. Pruneri, A. Vingiani, S. Demaria, F. Symmans, P. Nuciforo, L. Comerma, E.A. Thompson, S. Lakhani, S.R. Kim, S. Schnitt, C. Colpaert, C. Sotiriou, S.J. Scherer, M. Ignatiadis, S. Badve, R.H. Pierce, G. Viale, N. Sirtaine, F. PenaultLlorca, T. Sugie, S. Fineberg, S. Paik, A. Srinivasan, A. Richardson, Y. Wang, E. Chmielik, J. Brock, D.B. Johnson, J. Balko, S. Wienert, V. Bossuyt, S. Michiels, N. Ternes, N. Burchardi, S.J. Luen, P. Savas, F. Klauschen, P.H. Watson, B.H. Nelson, C. Criscitiello, S. O'Toole, D.

Larsimont, R. de Wind, G. Curigliano, F. Andre, M. Lacroix-Triki, M. van de Vijver, F. Rojo, G. Floris, S. Bedri, J. Sparano, D. Rimm, T. Nielsen, Z. Kos, S. Hewitt, B. Singh, G. Farshid, S. Loibl, K.H. Allison, N. Tung, S. Adams, K. Willard-Gallo, H.M. Horlings, L. Gandhi, A. Moreira, F. Hirsch, M.V. Dieci, M. Urbanowicz, I. Brcic, K. Korski, F. Gaire, H. Koeppen, A. Lo, J. Giltnane, M.C. Rebelatto, K.E. Steele, J. Zha, K. Emancipator, J.W. Juco, C. Denkert, J. Reis-Filho, S. Loi, S.B. Fox, Assessing Tumor-infiltrating Lymphocytes in Solid Tumors: A Practical Review for Pathologists and Proposal for a Standardized Method From the International Immunooncology Biomarkers Working Group: Part 1: Assessing the Host Immune Response, TILs in Invasive Breast Carcinoma and Ductal Carcinoma In Situ, Metastatic Tumor Deposits and Areas for Further Research, Adv Anat Pathol 24(5) (2017) 235-251.

[24] C. Denkert, S. Wienert, A. Poterie, S. Loibl, J. Budczies, S. Badve, Z. Bago-Horvath, A. Bane, S. Bedri, J. Brock, E. Chmielik, M. Christgen, C. Colpaert, S. Demaria, G. Van den Eynden, G. Floris, S.B. Fox, D. Gao, B. Ingold Heppner, S.R. Kim, Z. Kos, H.H. Kreipe, S.R. Lakhani, F. Penault-Llorca, G. Pruneri, N. Radosevic-Robin, D.L. Rimm, S.J. Schnitt, B.V. Sinn, P. Sinn, N. Sirtaine, S.A. O'Toole, G. Viale, K. Van de Vijver, R. de Wind, G. von Minckwitz, F. Klauschen, M. Untch, P.A. Fasching, T. Reimer, K. Willard-Gallo, S. Michiels, S. Loi, R. Salgado, Standardized evaluation of tumor-infiltrating lymphocytes in breast cancer: results of the 
ring studies of the international immuno-oncology biomarker working group, Mod Pathol 29(10) (2016) 1155-64.

[25] M.C. Wouters, F.L. Komdeur, H.H. Workel, H.G. Klip, A. Plat, N.M. Kooi, G.B. Wisman, M.J. Mourits, H.J. Arts, M.H. Oonk, R. Yigit, S. de Jong, C.J. Melief, H. Hollema, E.W. Duiker, T. Daemen, M. de Bruyn, H.W. Nijman, Treatment Regimen, Surgical Outcome, and T-cell Differentiation Influence Prognostic Benefit of Tumor-Infiltrating Lymphocytes in High-Grade Serous Ovarian Cancer, Clin Cancer Res 22(3) (2016) 714-24.

[26] C. Cortes, V. Vapnik, Support-Vector Networks, Machine Learning 20(3) (1995) 273-297. [27] Y.K. Lai, P.L. Rosin, Efficient circular thresholding, IEEE Trans Image Process 23(3) (2014) 992-1001.

[28] N. Malpica, C.O. de Solorzano, J.J. Vaquero, A. Santos, I. Vallcorba, J.M. Garcia-Sagredo, F. del Pozo, Applying watershed algorithms to the segmentation of clustered nuclei, Cytometry 28(4) (1997) 289-97.

[29] J. Wu, P. Zeng, Y. Zhou, C. Olivier, A novel color image segmentation method and its application to white blood cell image analysis, Proc. Int. Conf. Signal Process. 2 (2006) 16-20. [30] N. Otsu, A threshold selection method from gray level histograms., IEEE Trans. Syst. Man Cyber (9) (1979) 62-66.

[31] S.H. Rezatofighi, H. Soltanian-Zadeh, Automatic recognition of five types of white blood cells in peripheral blood, Comput Med Imaging Graph 35(4) (2011) 333-43.

[32] M. Saraswat, K.V. Arya, H. Sharma, Leukocyte segmentation in tissue images using differential evolution algorithm, Swarm and Evolutionary Computation 11 (2013) 46-54.

[33] L.C. Chen, G. Papandreou, I. Kokkinos, K. Murphy, A.L. Yuille, DeepLab: Semantic Image Segmentation with Deep Convolutional Nets, Atrous Convolution, and Fully Connected CRFs , IEEE Trans. Pattern Anal. Mach. Intell. 40(4) (2018) 834-848.

[34] M.D. Zeiler, R. Fergus, Visualizing and Understanding Convolutional Networks, Computer Vision - ECCV 2014 - 13th European Conference, Proceedings, Part I

, Zurich, Switzerland, 2014, pp. 818-833.

[35] S. Wienert, D. Heim, K. Saeger, A. Stenzinger, M. Beil, P. Hufnagl, M. Dietel, C. Denkert, F. Klauschen, Detection and segmentation of cell nuclei in virtual microscopy images: a minimum-model approach, Sci Rep 2 (2012) 503.

[36] S. Wienert, D. Heim, M. Kotani, B. Lindequist, A. Stenzinger, M. Ishii, P. Hufnagl, M. Beil, M. Dietel, C. Denkert, F. Klauschen, CognitionMaster: an object-based image analysis framework, Diagn Pathol 8 (2013) 34.

[37] R. Salgado, C. Denkert, S. Demaria, N. Sirtaine, F. Klauschen, G. Pruneri, S. Wienert, G. Van den Eynden, F.L. Baehner, F. Penault-Llorca, E.A. Perez, E.A. Thompson, W.F. Symmans, A.L. Richardson, J. Brock, C. Criscitiello, H. Bailey, M. Ignatiadis, G. Floris, J. Sparano, Z. Kos, T. Nielsen, D.L. Rimm, K.H. Allison, J.S. Reis-Filho, S. Loibl, C. Sotiriou, G. Viale, S. Badve, S. Adams, K. Willard-Gallo, S. Loi, T.W.G. International, The evaluation of tumor-infiltrating lymphocytes (TILs) in breast cancer: recommendations by an International TILs Working Group 2014, Ann Oncol 26(2) (2015) 259-71.

[38] A. Heindl, I. Sestak, K. Naidoo, J. Cuzick, M. Dowsett, Y. Yuan, Relevance of Spatial Heterogeneity of Immune Infiltration for Predicting Risk of Recurrence After Endocrine Therapy of ER+ Breast Cancer, J Natl Cancer Inst 110(2) (2018).

[39] S. Mika, C. Schaefer, P. Laskov, D. Tax, K.R. Muller, Support Vector Machines, 2004. [40] S. Haykin, Neural Networks and Learning Machines, Pearson2009.

[41] A. Janowczyk, A. Madabhushi, Deep learning for digital pathology image analysis: A comprehensive tutorial with selected use cases, J Pathol Inform 7 (2016) 29. 
[42] L. Ladicky, C. Russell, P. Kohli, P.H.S. Torr, Associative hierarchical CRFs for object class image segmentation, (2009) 739-746.

[43] O. Ronneberger, P. Fischer, T. Brox, U-Net: Convolutional Networks for Biomedical Image Segmentation, arXiv CoRR abs/1505.04597 (2015).

[44] E. Shelhamer, J. Long, T. Darrell, Fully Convolutional Networks for Semantic Segmentation, IEEE Trans. Pattern Anal. Mach. Intell. 39(4) (2017) 640-651.

[45] F. Sheikhzadeh, R.K. Ward, D. van Niekerk, M. Guillaud, Automatic labeling of molecular biomarkers of immunohistochemistry images using fully convolutional networks, PLoS One 13(1) (2018) e0190783.

[46] R. Bidart, M.J. Gangeh, M. Peikari, S. Salama, S. Nofech-Mozes, A.L. Martel, A. Ghodsi, Localization and classification of cell nuclei in post-neoadjuvant breast cancer surgical specimen using fully convolutional networks, Proc. SPIE 10581 (2018) 10581-10588.

[47] R.B. Girshick, Fast R-CNN, arXiv CoRR abs/1504.08083 (2015).

[48] S. Ren, K. He, R.B. Girshick, J. Sun, Faster R-CNN: Towards Real-Time Object Detection with Region Proposal Networks

, Advances in Neural Information Processing Systems 28: Annual Conference on Neural Information Processing Systems 2015

, Montreal, Quebec, Canada, 2015, pp. 91-99.

[49] J. Redmon, S.K. Divvala, R.B. Girshick, A. Farhadi, You Only Look Once: Unified, RealTime Object Detection, IEEE Conference on Computer Vision and Pattern Recognition, CVPR 2016.

, Las Vegas, NV, USA, 2016, pp. 779-788.

[50] J. Zhao, M. Zhang, Z. Zhou, J. Chu, F. Cao, Automatic detection and classification of leukocytes using convolutional neural networks, Med Biol Eng Comput 55 (2017) 1287-1301. [51] J. Chen, C. Srinivas, Automatic Lymphocyte Detection in H\{\\&\}E Images with Deep Neural

Networks, arXiv CoRR abs/1612.03217 (2016).

[52] E. Garcia, R. Hermoza, C.B. Castanon, L. Cano, M. Castillo, C. Castanneda, Automatic Lymphocyte Detection on Gastric Cancer IHC Images Using Deep Learning, 2017 IEEE 30th International Symposium on Computer-Based Medical Systems (CBMS) (2017) 200-204. [53] T. Chen, C. Chefd'hotel, Deep Learning Based Automatic Immune Cell Detection for Immunohistochemistry Images, Machine Learning in Medical Imaging (2014) 17-24.

[54] Z. Swiderska-Chadaj, H. Pinckaers, M. van Rijthoven, M. Balkenhol, M. Melnikova, O. Geessink, Q.F. Manson, G. Litjens, J. van der Laak, F. Ciompi, Convolutional Neural Networks for Lymphocyte detection in Immunohistochemically Stained Whole-Slide Images, 2018. [55] S. Bach, A. Binder, G. Montavon, F. Klauschen, K.R. Muller, W. Samek, On Pixel-Wise Explanations for Non-Linear Classifier Decisions by Layer-Wise Relevance Propagation, PLoS One 10(7) (2015) e0130140.

[56] G. Montavon, S. Lapuschkin, A. Binder, W. Samek, K.R. Müller, Explaining nonlinear classification decisions with deep taylor decomposition, Pattern Recognition 65 (2017) 211222.

[57] A. Binder, M. Bockmayr, M. Haegele, S. Wienert, D. Heim, K. Hellweg, A. Stenzinger, L. Parlow, J. Budczies, B. Goeppert, D. Treue, M. Kotani, M. Ishii, M. Dietel, A. Hocke, C. Denkert, K.R. Muller, F. Klauschen, Towards computational fluorescence microscopy: Machine learning-based integrated prediction of morphological and molecular tumor profiles, ArXiv e-prints (2018) 1805.11178.

[58] K. Simonyan, A. Zisserman, Very Deep Convolutional Networks for Large-Scale Image Recognition, arXiv/CoRR abs/1409.1556 (2014). 
[59] G. Huang, Z. Liu, K.Q. Weinberger, Densely Connected Convolutional Networks, IEEE Conference on Computer Vision and Pattern Recognition (2017) 2261-2269.

[60] G. Montavon, W. Samek, K.R. Muller, Methods for interpreting and understanding deep neural networks, Digital Signal Processing 73 (2018) 1-15.

[61] L. Shi, R. Kusko, R.D. Wolfinger, B. Haibe-Kains, M. Fischer, S.A. Sansone, C.E. Mason, C. Furlanello, W.D. Jones, B. Ning, W. Tong, The international MAQC Society launches to enhance reproducibility of high-throughput technologies, Nat Biotechnol 35(12) (2017) 1127-1128.

[62] A.M. Newman, C.L. Liu, M.R. Green, A.J. Gentles, W. Feng, Y. Xu, C.D. Hoang, M. Diehn, A.A. Alizadeh, Robust enumeration of cell subsets from tissue expression profiles, Nat Methods 12(5) (2015) 453-7.

[63] E. Becht, N.A. Giraldo, L. Lacroix, B. Buttard, N. Elarouci, F. Petitprez, J. Selves, P. Laurent-Puig, C. Sautes-Fridman, W.H. Fridman, A. de Reynies, Estimating the population abundance of tissue-infiltrating immune and stromal cell populations using gene expression, Genome Biol 17(1) (2016) 218.

[64] H.R. Ali, L. Chlon, P.D. Pharoah, F. Markowetz, C. Caldas, Patterns of Immune Infiltration in Breast Cancer and Their Clinical Implications: A Gene-Expression-Based Retrospective Study, PLoS Med 13(12) (2016) e1002194.

[65] A.J. Gentles, A.M. Newman, C.L. Liu, S.V. Bratman, W. Feng, D. Kim, V.S. Nair, Y. Xu, A. Khuong, C.D. Hoang, M. Diehn, R.B. West, S.K. Plevritis, A.A. Alizadeh, The prognostic landscape of genes and infiltrating immune cells across human cancers, Nat Med 21(8) (2015) 938-945.

[66] M.A. Laginestra, C. Tripodo, C. Agostinelli, G. Motta, S. Hartmann, C. Doring, M. Rossi, F. Melle, M.R. Sapienza, V. Tabanelli, A. Pileri, F. Fuligni, A. Gazzola, C. Mannu, C.A. Sagramoso, S. Lonardi, L. Lorenzi, F. Bacci, E. Sabattini, A. Borges, I. Simonitsch-Klupp, J. Cabecadas, E. Campo, J. Rosai, M.L. Hansmann, F. Facchetti, S.A. Pileri, Distinctive Histogenesis and Immunological Microenvironment Based on Transcriptional Profiles of Follicular Dendritic Cell Sarcomas, Mol Cancer Res 15(5) (2017) 541-552.

[67] J. Jeschke, M. Bizet, C. Desmedt, E. Calonne, S. Dedeurwaerder, S. Garaud, A. Koch, D. Larsimont, R. Salgado, G. Van den Eynden, K. Willard Gallo, G. Bontempi, M. Defrance, C. Sotiriou, F. Fuks, DNA methylation-based immune response signature improves patient diagnosis in multiple cancers, J Clin Invest 127(8) (2017) 3090-3102.

[68] D.J. McGrail, L. Federico, Y. Li, H. Dai, Y. Lu, G.B. Mills, S. Yi, S.Y. Lin, N. Sahni, Multiomics analysis reveals neoantigen-independent immune cell infiltration in copy-number driven cancers, Nat Commun 9(1) (2018) 1317.

[69] Y. Yuan, H. Failmezger, O.M. Rueda, H.R. Ali, S. Graf, S.F. Chin, R.F. Schwarz, C. Curtis, M.J. Dunning, H. Bardwell, N. Johnson, S. Doyle, G. Turashvili, E. Provenzano, S. Aparicio, C. Caldas, F. Markowetz, Quantitative image analysis of cellular heterogeneity in breast tumors complements genomic profiling, Sci Transl Med 4(157) (2012) 157ra143.

[70] J. Saltz, R. Gupta, L. Hou, T. Kurc, P. Singh, V. Nguyen, D. Samaras, K.R. Shroyer, T. Zhao, R. Batiste, J. Van Arnam, N. Cancer Genome Atlas Research, I. Shmulevich, A.U.K. Rao, A.J. Lazar, A. Sharma, V. Thorsson, Spatial Organization and Molecular Correlation of TumorInfiltrating Lymphocytes Using Deep Learning on Pathology Images, Cell Rep 23(1) (2018) 181-193 e7.

[71] U. Djuric, G. Zadeh, K. Aldape, P. Diamandis, Precision histology: how deep learning is poised to revitalize histomorphology for personalized cancer care, npj Precision Oncology 1(1) (2017). 